\title{
Magnitude and Associated Factors of Post Spinal Hypotension Among Pregnant Mothers Who Delivered by Elective Caesarean Section at Gandhi Memorial Hospital, Addis Ababa, Ethiopia
}

\author{
Ashebir Nigussie Yirgu ${ }^{1,}$, Wosenyeleh Admasu Sahile ${ }^{2}$, Abdurahman Tune Dedecho ${ }^{1}$, \\ Mohammed Suleiman Obsa ${ }^{3}$, Zewde Zema Kanche ${ }^{4}$ \\ ${ }^{1}$ Department of Anesthesia, Arsi University, Asella, Ethiopia \\ ${ }^{2}$ Department of Anesthesia, Addis Ababa University, Addis Ababa, Ethiopia \\ ${ }^{3}$ Department of Anesthesia, Wolaita Sodo University, Wolaita, Ethiopia \\ ${ }^{4}$ Department of Pharamcy, Wolaita Sodo University, Wolaita, Ethiopia
}

Email address:

Ashebirnigussie3@gmail.com (A. N. Yirgu)

*Corresponding author

To cite this article:

Ashebir Nigussie Yirgu, Wosenyeleh Admasu Sahile, Abdurahman Tune Dedecho, Mohammed Suleiman Obsa, Zewde Zema Kanche. Magnitude and Associated Factors of Post Spinal Hypotension Among Pregnant Mothers Who Delivered by Elective Caesarean Section at Gandhi Memorial Hospital, Addis Ababa, Ethiopia. Clinical Medicine Research. Vol. 9, No. 4, 2020, pp. 85-90. doi: $10.11648 /$ j.cmr.20200904.13

Received: June 17, 2020; Accepted: July 21, 2020; Published: August 10, 2020

\begin{abstract}
Introduction: Hypotension is a $20 \%$ to $30 \%$ reduction in baseline blood pressure. Infrequently, hypotension after spinal anesthesia can be significantly severe in pregnant mother and increases intraoperative and postoperative morbidity and mortality. Objectives: To assess magnitude and associated factors of post spinal hypotension among pregnant mothers who delivered by elective cesarean sections from January to February 2016 at Gandhi Memorial Hospital, Addis Ababa. Methods: Institution based cross sectional study design was conducted. All lists of pregnant mothers who gave birth by elective cesarean section under spinal anesthesia at Gandhi Memorial Hospital were included. Training was given for data collectors and supervisors. Regular supervision and follow up were made. Data was entered in to Epi info version 7 software and transported to SPSS version 20 for analysis. Odd ratio and $95 \%$ confidence interval was computed. Bivariate and Multivariate analysis used to identify factors associated with hypotension. Results: The study was conducted on 60 study participants. 24 (40\%) were equally found between 25-29 years and 30-34 years. The incidence of hypotension was $80 \%$ during five to fifteen minute and $83 \%$ during fifteen to twenty five minute. $70 \%$ of the study participants were preloaded with more than $500 \mathrm{ml}$ of crystalloid and $60 \%$ were took oxytocin for uterine contraction. Majority of them were in supine position prior to spinal anesthesia. Conclusion and recommendation: The magnitude of post spinal hypotension was so high that the use vigorous fluid resuscitation and the use of prophylactic vasopressor were recommended.
\end{abstract}

Keywords: Hypotension, Pre-loading, Spinal Anesthesia

\section{Introduction}

Post spinal hypotension is a common physiological complication with an incidence ranging from $25-75 \%$ among general population in the world and higher in patients undergoing caesarean section $[1,2]$. The most common definitions of hypotension after spinal anesthesia for caesarean delivery is a $20 \%$ to $30 \%$ reduction baseline systolic blood pressure [3]. up to $80 \%$ of anesthetist require the use of vasopressor to treat post spinal hypotension [4].

It is mainly occurred due to sympathetic blockade leading to peripheral vasodilatation and venous pooling of blood. As a result, there is decreased venous return and cardiac output leading to hypotension [5]. The risk of hypotension is 
increased when there is higher level of block [6].

Preloading before spinal anesthesia reduces the incidence of hypotension in the first 5 minutes [7]. However, colloid is more dependable than crystalloid to prevent hypotension [1].

Hypotension occurs less frequently when spinal anesthesia is performed in lateral position as compared to sitting position [8]. Staying patient in the sitting position for 5 minutes' results in lower sensory block levels that results in decrease in venous return and requirement of ephedrine for the treatment of hypotension following hyperbaric bupivacaine [9]. In addition, slower speed of injection resulted in a lower incidence of hypotension and in addition, the hypotension was of delayed onset, shorter duration and required less ephedrine [10].

The aim of the study is to collect risk factors of post spinal hypotension and its prevalence so as to provide a baseline data and to encourage preventive methods of hypotension following caesarian deliveries under spinal anesthesia.

\section{Method and Materials}

Study area and period

The study was conducted at Gandhi Memorial Hospital located in Addis Ababa which is capital city of Ethiopia. It was established in $1958 \mathrm{G}$. C when it was called the only maternity hospital in Ethiopia. The hospital was named as Gandhi Memorial Hospital for the memory of Mohatema Gandhi. It is one of the thirteen governmental hospitals found in Addis Ababa and one of the hospitals which was administered by Addis Ababa Health Bureau. The Hospital primarily gives services for women and children. The Hospital provides Gynecologic, Obstetric and reproductive health services including Mother and Child Health $(\mathrm{MCH})$, infertility and sexual violence services. Currently, it is providing inpatient, outpatient services and emergency cases. In October 2016, the hospital established a neonatal unit and currently serves as inpatient unit for at least 120 neonates per month. The hospital has 110 beds and delivers 25 neonates each day. The hospital has four operation theatre and average number of elective caesarian deliveries done at the hospital is two per day. Study was conducted from January to February 2016.

Study design: -Cross sectional study design was employed.

Source population: all pregnant mothers who gave birth by elective caesarian section at Gandhi Memorial Hospital, Addis Ababa, Ethiopia, 2016.

Study population: Selected pregnant mothers who gave birth by elective caesarian section from January to February at Gandhi Memorial Hospital, Addis Ababa, Ethiopia, 2016.

Exclusion criteria: Failed spinal anesthesia, mothers who take antihypertensive drugs on the day of surgery and Preoperative maternal hypotension.

Sampling technique and sample size determination: All list of mothers who come for elective cesarean section and done under spinal anesthesia from January to February 2016 at Gandhi Memorial Hospital was included. so that sampling technique and sample size determination was not used.

Data collection tools and procedure: Data was collected using pretested structured questionnaires. It was prepared in English and translated to Amharic and again back translation to English was made to check for consistency. Data was collected by two BSC holder anesthetists and supervised by one MSC holder Anesthetist.

Data Quality Assurance: Data collectors and supervisors was trained for a half day on each items included in the study tools, objective, relevant of study, right of respondents, confidentiality of information obtained. During data collection, regular supervision and follow up was made. Investigator was check for completeness and consistency of data on daily basis. Once the data was collected and checked for completeness, consistency and accuracy, it was sorted, categorized and summarized. Double entry was made on $10 \%$ of the collected population.

Data Analyzing and processing

The data was entered on epi info version 7 and was exported to SPSS version 20 computer program for analysis. Descriptive statistics was used to summarize data, tables and figures for display results. Bivariate and multivariate analysis was used to see the effect of independent variable on post spinal hypotension. Variables which were significant on bivariate analysis at p-value less than 0.2 was taken to multivariate analysis. In multivariate analysis $\mathrm{P}$ - value of less than 0.05 was used as a cut of point for presence of association. Strength of association was measured by $95 \%$ confidence interval and/ Odd ratio.

Ethical Consideration

Ethical clearance and approval was obtained from ethical review committee, Anesthesia department, Addis Ababa University. Permission to conduct was obtained from Gandhi memorial Hospital. Informed written consent was secured from every study participant before the start of the interview after telling them about the objective of the study. The obtained data was only used for study purpose. Confidentiality and anonymity were ensured.

Operational Definition

Spinal anesthesia: injecting small amounts of local anesthetic into the cerebro-spinal fluid (CSF).

Post spinal hypotension: hypotension occurs after administration of local anesthetics.

Cesarean delivery: delivery of a fetus by surgical incisions made through the abdominal wall and the uterine wall.

Elective caesarean delivery: caesarean delivery done before the onset of labor.

Hypotension is Systolic blood pressures less than $85-90$ $\mathrm{mm} \mathrm{Hg}$ or a decrease of more than $20 \%-30 \%$ from the baseline value.

Mild hypotension is a decrease of mean arterial pressure $20 \%$ below the baseline

Moderate hypotension is a decrease of mean arterial pressure to below $20 \%$ to $30 \%$ from the base line

Severe hypotension is a decrease of mean arterial pressure greater than $30 \%$ below the baseline.

Hypertension is a Systolic blood pressure greater than 140 $\mathrm{mm} \mathrm{Hg}$ or an increase of more than $20 \%-30 \%$ from the preanesthetic value. 
Multiple gestations is a pregnancy condition carrying two or more fetuses simultaneously

Gravidity is the number of times a woman has been pregnant, including current pregnancy.

Position of the patient is the pregnant mother's position prior to spinal anesthesia and during spinal anesthesia is given.

Duration of crystalloid loading is the time taken from the beginning of the preloading to start of spinal anesthesia

Amount of crystalloid loading is the amount of fluid administered before SA.

\section{Result}

Socio-demographic characteristics

Total number of pregnant mothers included in the study was 60. Among them, the highest number 24 (40\%) were belonged equally to the age group of $25-29$ years and 30-34 years. The mean age of respondents was 29.30 (minimum 22 and maximum 35). 24 (39.3\%) were House wife. Half of the total participants are Amhara and overweight by body mass index (table 1).

Change in blood pressure

Comparing change in blood pressure from 5 to 15 minute to 15 to 25 minute, majority of pregnant mother developed moderate hypotension in the 15 to 25 minute. About 24 of all cases had mild hypotension from 5 to 15 minute. In both time groups equal number $(n=4)$ of pregnant mothers develop severe hypotension. None of the patients used prophylactic vasopressor and colloid. (Figure 1)

Table 1. Socio-demographic characteristics of pregnant mothers who gave birth by elective ceserian section under spinal anesthesia at Gandhi Memorial Hospital, Addis Ababa, Ethiopia, 2016.

\begin{tabular}{llll}
\hline Variables & Category & Frequency & Percent \\
\hline \multirow{4}{*}{ Age } & $20-24$ & 6 & 10.0 \\
& $25-29$ & 24 & 40.0 \\
& $30-34$ & 24 & 40.0 \\
& $35-39$ & 6 & 10.0 \\
BMI & Total & 60 & 100.0 \\
& $18.5-24.5$ & 18 & 30.0 \\
& $25-29.5$ & 30 & 50.0 \\
Ethnicity & $30-34.5$ & 12 & 20.0 \\
& Total & 60 & 100.0 \\
& Amhara & 30 & 50.0 \\
& Oromo & 24 & 40.0 \\
& Other & 6 & 10.0 \\
& Total & 60 & 100.0 \\
& Engineer & 6 & 9.8 \\
& federal police & 6 & 9.8 \\
& house wife & 24 & 39.3 \\
& Merchant & 6 & 9.8 \\
& Police & 6 & 9.8 \\
& Secretary & 6 & 9.8 \\
& Teacher & 6 & 9.8 \\
& Total & 60 & 100.0 \\
\hline
\end{tabular}

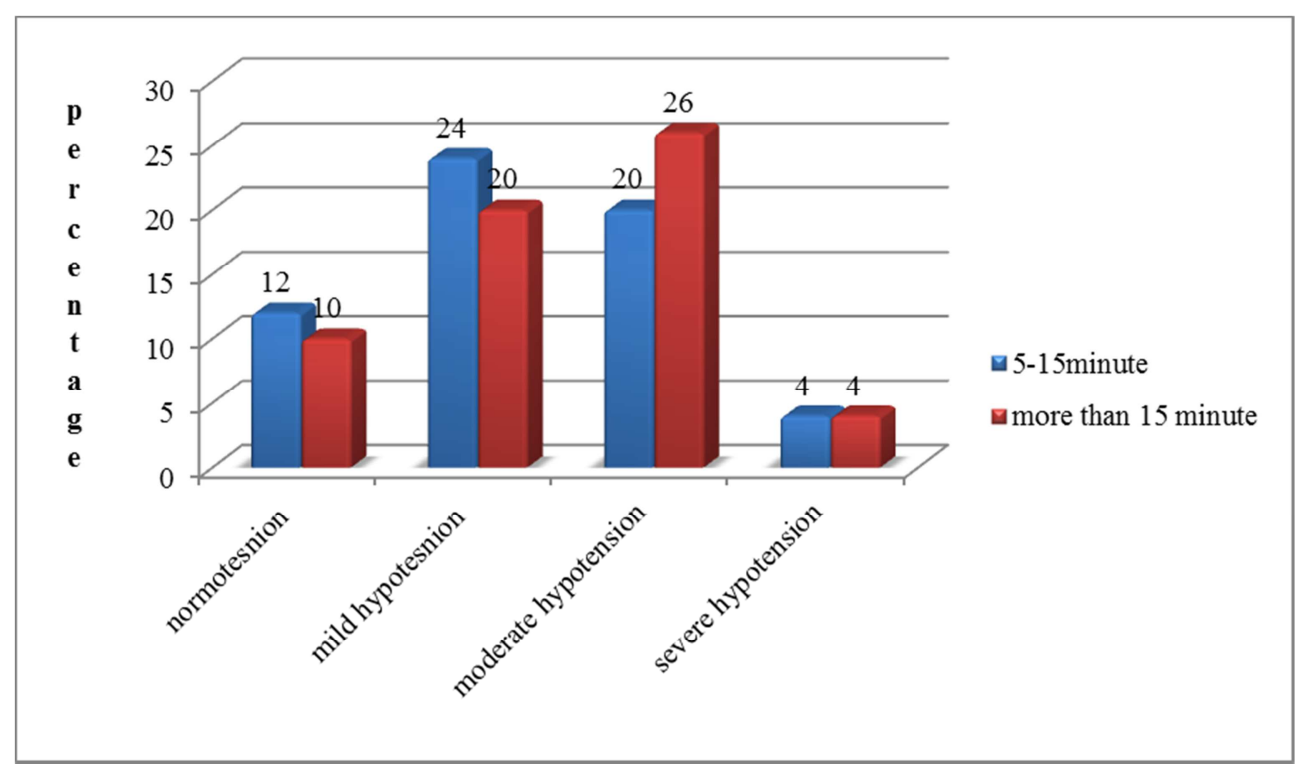

Figure 1. Change in the blood pressure after spinal anesthesia in pregnant mothers who gave birth by elective ceserian section under spinal anesthesia in Gandhi Memorial Hospital, Addis Ababa, Ethiopia, 2016.

Associated factors of post spinal hypotension between 515 minute

Amount of fluid preloading $(>500 \mathrm{ml})$, duration of crystalloid loading before spinal, BMI was strongly associated with post spinal hypotension at p-value less than 0.05 at five to fifteen-minute post spinal anesthesia while Gestational age in terms, types of gestation pregnancy, Patient positioning prior to SA, type of uterotonic agent used were not associated with post spinal hypotension at the same value. The mothers who took less than or equal to $500 \mathrm{ml}$ fluid preloading developing post spinal hypotension 2 times more than who took more than $500 \mathrm{ml}$ fluid. (AOR, 1.897 $95 \%$ C. I, 0.246 - 3.272). The pregnant mother who was preloading with crystalloid for greater than $20 \mathrm{~min}$ before spinal anesthesia was about 9 times more likely to develop hypotension than those preloaded for less than or equal to 20 min before spinal anesthesia. (AOR, 9.000, 95\% C. I, 1.418 57.117). (Table 2) 
Table 2. Association of factors affecting post spinal anesthesia at five to fifteen minute after spinal anesthesia in pregnant mothers who gave birth by elective caesarian section under spinal anesthesia in Gandhi Memorial Hospital, Addis Ababa, Ethiopia, 2016.

\begin{tabular}{|c|c|c|c|c|c|}
\hline \multirow{2}{*}{ Variable } & \multirow{2}{*}{ Category } & \multirow{2}{*}{ P-Value } & \multirow{2}{*}{ AOR } & \multicolumn{2}{|c|}{ 95\% C. I. for EXP (B) } \\
\hline & & & & Lower & Upper \\
\hline \multirow{3}{*}{ Gestational age in terms } & Preterm & $*$ & $*$ & * & $*$ \\
\hline & Term & .390 & .324 & .025 & 4.230 \\
\hline & Post term & .619 & .686 & .156 & 3.026 \\
\hline \multirow{2}{*}{ Types of gestation pregnancy } & Single & .221 & .399 & * & * \\
\hline & Multiple & 1.547 & .520 & 0.475 & 1.587 \\
\hline \multirow{2}{*}{ BMI } & Normal & * & * & * & * \\
\hline & Over weight & .028 & .798 & .653 & .976 \\
\hline \multirow{2}{*}{ Patient positioning prior to SA } & Supine & .746 & .778 & .170 & 3.564 \\
\hline & Left lateral & * & * & * & * \\
\hline \multirow{3}{*}{ Type of uterotonic agent used } & Oxytocin & .975 & .974 & .186 & 5.096 \\
\hline & Ergometrine & .217 & .174 & .011 & 2.783 \\
\hline & Both & * & * & * & * \\
\hline \multirow{2}{*}{ Duration of crystalloid loading before spinal } & $<=20 \min$ & $*$ & $*$ & * & * \\
\hline & $>20 \mathrm{~min}$ & 0.020 & 9.000 & 1.418 & 57.117 \\
\hline \multirow{2}{*}{ Amount of fluid preloading } & $<=500 \mathrm{ml}$ & .870 & 1.897 & .246 & 3.272 \\
\hline & $>500 \mathrm{ml}$ & * & $*$ & $*$ & * \\
\hline
\end{tabular}

Associated factors of post spinal hypotension between $15-$ 25 minute

BMI, Oxytocin agent used, amount of fluid preloading was strongly associated with post spinal hypotension at p-value less than 0.05 at fifteen minute to twenty-five minutes while Gestational age in terms, types of gestation pregnancy, Patient positioning prior to Spinal anesthesia and duration of crystalloid loading were not associated with post spinal hypotension at the same value. The mothers who took oxytocin develop post spinal hypotension 4 times more than those who took ergometrine or both. (AOR, 3.965 95\% C. I, 1.473-10.674). The pregnant mother who placed in supine position prior to spinal anesthesia was about 3 times more likely to develop hypotension than left lateral position. (AOR, 2.953 95\% C. I, 0.356 - 24.519). (Table 3)

Table 3. Association of factors affecting post spinal anesthesia at fifteen to twenty-five minute after spinal anesthesia in pregnant mothers who gave birth by elective caesarian section under spinal anesthesia in Gandhi Memorial Hospital, Addis Ababa, Ethiopia, 2016.

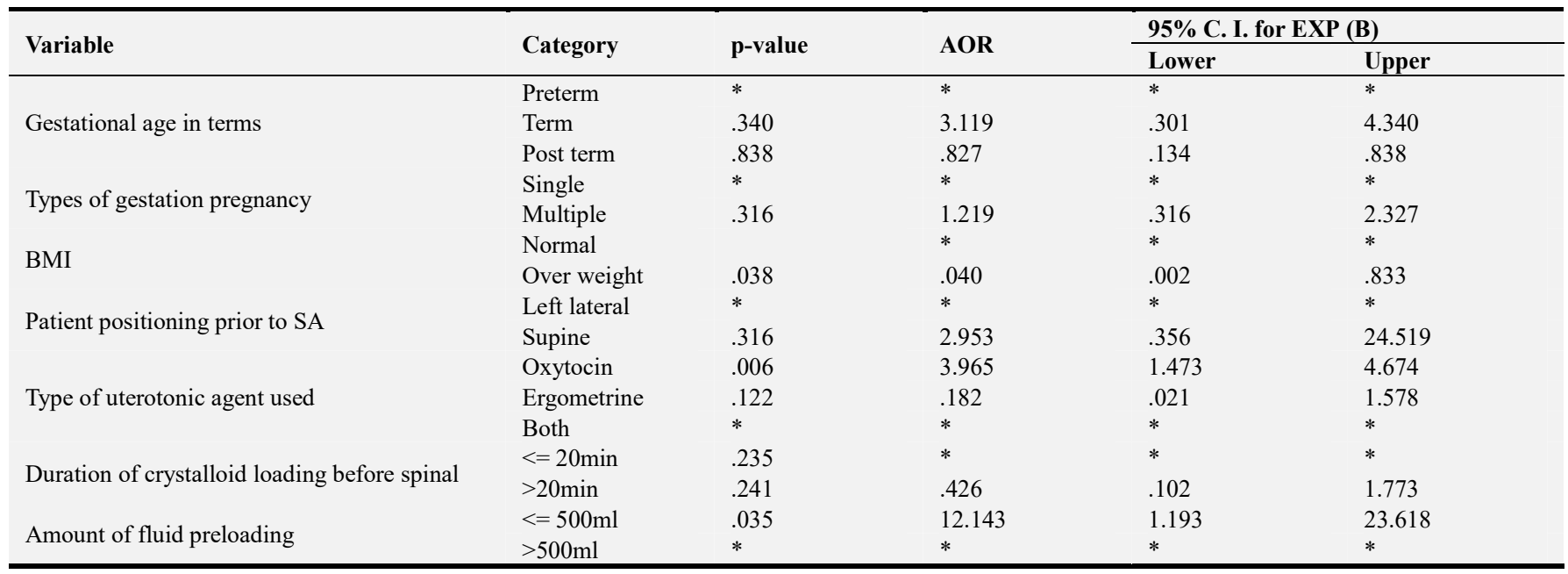

\section{Discussion}

Most of the pregnant mother were belonged similarly to the age group of 25-29 year 24 (40\%) and age group of 30-34 year $24(40 \%)$. The mean average is 29.30 (minimum 22 and maximum 35). Incidence of post spinal hypotension was $80 \%$ in five to fifteen minutes and $83.7 \%$ in fifteen to twenty-five minutes. The result from University Bangkok, Thailand, revealed that the incidence of post spinal hypotension was $52.6 \%$ [11]. This is most likely due to a pre-load fluid bolus are not sufficient to maintain hemodynamic stability after the initiation of spinal anesthesia and they used vasopressor as prophylaxis and add adjuvant drugs.

The present study indicated that the highest decrease in Systolic blood pressure occurred in both time groups after spinal anesthesia which is consistent with other similar studies $[11,12]$. This can be due to the sensitivity of the autonomous nervous system and sympathetic paralysis below the blockage site before sensory and motor paralysis. Moreover, in pregnant women, the hypotension can also be result of aortic and inferior vena cava compression in supine 
position after spinal anesthesia [13].

This study revealed that amount of fluid preloading $(<500 \mathrm{ml})$ and duration of crystalloid loading before spinal anesthesia was strongly associated with post spinal hypotension at five to fifteen minutes. And amount of fluid preloading $(<500 \mathrm{ml})$ was also associated with post spinal hypotension from fifteen to twenty-five minutes. The odd of developing post spinal hypotension was two times more in mothers who took less than or equal to $500 \mathrm{ml}$ fluid preloading than the odd of developing post spinal hypotension who took more than $500 \mathrm{ml}$ fluid. However, another study Rout et al, noted that the incidence of hypotension was reduced in patients who received crystalloid $20 \mathrm{ml} / \mathrm{kg}$ than in patients without pre-hydration [14].

In this study, the pregnant mother who was preloading with crystalloid for greater than $20 \mathrm{~min}$ before spinal anesthesia was about 9 times more likely to develop hypotension than those preloaded for less than or equal to 20 minutes. In contrast, a study done in South Korea, Department of Anesthesiology and pain Medicine, the timing of crystalloid loading does not have an impact on the incidence of post spinal hypotension [15]. This is most likely due to only small portion of crystalloid fluid is remained in intravascular space at the time of vasodilatation after spinal anesthesia.

In the study, it was found that took oxytocin was four times more likely to develop post spinal hypotension than the pregnant mother who underwent spinal anesthesia and took ergometrine for uterine contraction from fifteen to twentyfive-minute post spinal anesthesia. Another study done in United Kingdom, Birmingham Women's Hospital suggested that, although there is controversial concerning the effect of oxytocin on hemodynamic effect, there is a decrease in mean arterial blood pressure in healthy women having elective caesarian section [16].

According to the findings of this study, the odds of developing post spinal hypotension in supine position prior to spinal anesthesia was three times more likely as compared to developing post spinal hypotension in left lateral position. The findings of other study revealed that the degree of hypotension was higher in supine when compared to left lateral position. During supine position the gravid uterus of the pregnant woman compresses the aorta and the inferior vena cava against the bodies of lumbar of vertebra. This results in decreased venous return which may decreases maternal cardiac output and blood pressure [17].

It was also observed from the result of current study that body mass index was significantly associated with the development of post spinal hypotension at both five to fifteen and fifteen to twenty-five minutes. Similarly, another studies have suggested that patients with an increased BMI are at increased risk for the development of hypotension under spinal anesthesia for Caesarean delivery [18].

\section{Conclusion}

In this study, the magnitude of post spinal hypotension was high at both time intervals. Body mass index, amount of fluid preloading and duration of crystalloid loaded before spinal anesthesia were the major factors of post spinal hypotension at both intervals. Preloading the pregnant mother with more than $500 \mathrm{ml}$ of crystalloid for 20 minute prior to spinal anesthesia to increase venous return, use of Ergometrine and combination of ergometrine and oxytocin to stabilize hemodynamic effects and position the patient in left lateral position prior to spinal anesthesia to avoid the effect of aortocaval compression caused by gravid uterus had a great impact to reduce the incidence of post spinal hypotension.

\section{Funding}

Addis Ababa University.

\section{Abbreviation}

AAU: Addis Ababa University; GMH; Gandhi Memorial Hospital; NVD: Normal Vaginal Delivery

\section{Competing Interests}

We declared that we had no competing interests.

\section{Authors' Contributions}

MS and AN has contributed to conception, design of the study, data supervision, data entry clerk, data analyses, result interpretation, manuscript development and revision. WA and ZZ contributed data analyses, result interpretation and manuscript development. All authors read and approve the final manuscript.

\section{Acknowledgements}

We would like to thank all the staff in the of GMH for providing valuable information.

\section{References}

[1] Morgan PJ, Halpern SH, Tarshis J (2001) The effects of an increase of central blood volume before spinal anesthesia for cesarean delivery: a qualitative systematic review. Anesthesia \& Analgesia 92: 997-1005.

[2] Bajwa SJS, Bajwa SK, Kaur J, Singh A, Singh A, et al. (2012) Prevention of hypotension and prolongation of postoperative analgesia in emergency cesarean sections: A randomized study with intrathecal clonidine. International journal of critical illness and injury science 2: 63 .

[3] Macarthur A (2002) Solving the problem of spinal-induced hypotension in obstetric anesthesia. Canadian Journal of Anesthesia/Journal canadien d'anesthésie 49: 536-539.

[4] Lee A, Kee WDN, Gin T (2002) A quantitative, systematic review of randomized controlled trials of ephedrine versus phenylephrine for the management of hypotension during spinal anesthesia for cesarean delivery. Anesthesia \& Analgesia 94: 920-926. 
[5] Ueyama H, He Y-L, Tanigami H, Mashimo T, Yoshiya I (1999) Effects of crystalloid and colloid preload on blood volume in the parturient undergoing spinal anesthesia for elective Cesarean section. The Journal of the American Society of Anesthesiologists 91: 1571-1571.

[6] Kee WDN (2010) Prevention of maternal hypotension after regional anaesthesia for caesarean section. Current Opinion in Anesthesiology 23: 304-309.

[7] Ewaldsson CA, Hahn R (2001) Volume kinetics of Ringer's solution during induction of spinal and general anaesthesia. British journal of anaesthesia 87: 406-414.

[8] Obasuyi B, Fyneface-Ogan S, Mato C (2013) A comparison of the haemodynamic effects of lateral and sitting positions during induction of spinal anaesthesia for caesarean section. International journal of obstetric anesthesia 22: 124-128.

[9] Loubert C, Hallworth S, Fernando R, Columb M, Patel N, et al. (2011) Does the baricity of bupivacaine influence intrathecal spread in the prolonged sitting position before elective cesarean delivery? A prospective randomized controlled study. Anesthesia \& Analgesia 113: 811-817.

[10] Simon L, Boulay G, Ziane A, Noblesse E, Mathiot J, et al. (2000) Effect of injection rate on hypotension associated with spinal anesthesia for cesarean section. International journal of obstetric anesthesia 9: 10-14.

[11] Reidy J, Douglas J (2008) Vasopressors in obstetrics. Anesthesiology clinics 26: 75-88.
[12] KlÖHR S, Roth R, Hofmann T, Rossaint R, Heesen M (2010) Definitions of hypotension after spinal anaesthesia for caesarean section: literature search and application to parturients. Acta Anaesthesiologica Scandinavica 54: 909-921.

[13] Dahlgren G, Granath F, Wessel H, Irestedt L (2007) Prediction of hypotension during spinal anesthesia for cesarean section and its relation to the effect of crystalloid or colloid preload. International journal of obstetric anesthesia 16: 128-134.

[14] Loubert C (2012) Fluid and vasopressor management for Cesarean delivery under spinal anesthesia: continuing professional development. Canadian Journal of Anesthesia/Journal canadien d'anesthésie: 1-16.

[15] Banerjee A, Stocche RM, Angle P, Halpern SH (2010) Preload or coload for spinal anesthesia for elective Cesarean delivery: a meta-analysis. Canadian Journal of Anesthesia/Journal canadien d'anesthésie 57: 24-31.

[16] Scrutton M (2004) Update in obstetric anaesthesia oxytocin: what dose and why. Anaesthesia Points West 37: 28-30.

[17] Kinsella S (2003) Lateral tilt for pregnant women: why 15 degrees? Anaesthesia 58: 835-836.

[18] Frölich MA, Caton D (2002) Baseline heart rate may predict hypotension after spinal anesthesia in prehydrated obstetrical patients. Canadian Journal of Anesthesia/Journal canadien d'anesthésie 49: 185-189. 